\title{
Safety and efficacy assessment of transarterial chemoembolization using drug-eluting beads in patients with hepatocellular carcinoma and arterioportal shunt: a single-center experience
}

This article was published in the following Dove Medical Press journal: Cancer Management and Research

\section{Yu-dong Xiao \\ Cong Ma \\ Zi-shu Zhang* \\ Jun Liu*}

Department of Radiology, The Second Xiangya Hospital of Central South University, Changsha 4I00II, China

*These authors contributed equally to this work
Correspondence: Zi-shu Zhang; Jun Liu Department of Radiology, The Second Xiangya Hospital of Central South University, No. 139 Middle Renmin Road, Changsha 4100II, China

Tel +86 I59 73II 60I7;

+86 13787085002

Email zishuzhang@csu.edu.cn; junliu123@csu.edu.cn

\begin{abstract}
Objective: To evaluate the feasibility and safety of transarterial chemoembolization with drugeluting beads (DEB-TACE) in patients with hepatocellular carcinoma (HCC) and arterioportal shunts (APSs).
\end{abstract}

Materials and methods: Fifty-eight patients with unresectable HCC and APSs who were treated with DEB-TACE ( $n=26$ ) or polyvinyl alcohol (PVA) plus TACE (PVA-TACE, $n=32$ ) were included in this retrospective study. The tumor response was evaluated by the modified Response Evaluation Criteria in Solid Tumors. Toxicity was graded by the Common Terminology Criteria for Adverse Events version 5.0 (CTCAE v5.0) and compared between the two groups. Survival curves were calculated by the Kaplan-Meier method and compared by the log-rank test between the two groups. The influence of potential prognostic factors on survival in the DEB-TACE group was analyzed via a multivariate Cox regression model.

Results: The disease control rate was better in the DEB-TACE group than in the PVA-TACE group. The median survival times were 346 and 274 days in the DEB-TACE group and PVATACE group, respectively. There was no significant difference in survival rates between the two groups $(P=0.081)$. Patients treated with DEB-TACE were significantly less likely to have fever $(P=0.048)$ or a low-grade (grade $1-2)$ increase in transaminases $(P=0.046)$ than the patients treated with PVA-TACE. The potential predictive prognostic factors in the DEB-TACE group were tumor response, APS grading, and serum bilirubin.

Conclusion: DEB-TACE may be feasible and safe in HCC patients with APS. Survival in the DEB-TACE group was associated with tumor response, APS grading, and serum bilirubin levels. Keywords: carcinoma, hepatocellular, chemoembolization, therapeutic, microspheres, arteriovenous fistula

\section{Introduction}

Arterioportal shunt (APS) is the redistribution of hepatic arterial flow into portal venous flow due to abnormal communication between the hepatic artery and portal vein and may result in portal hypertension, refractory ascites, bleeding of esophageal and gastric varices, and hepatic encephalopathy. ${ }^{1-4}$ It has been reported that the occurrence of APS is $28.8 \%-63.2 \%$ in hepatocellular carcinoma (HCC) patients. ${ }^{5,6}$ Hepatic APS can be involved through four different types of routes: transsinusoidal route, transvasal route, transplexal route, and transtumoral route. ${ }^{1}$ Each route can occur alone or in combination with other mechanisms. When performing conventional transarterial 
chemoembolization (cTACE), intra-arterial Lipiodol will pass through the APS into the portal vein, resulting in embolization of the normal liver. ${ }^{7-9}$ Therefore, it is crucial to use a suitable embolic material to avoid inadvertent embolization of the normal liver during TACE.

Drug-eluting beads (DEBs) are particulate embolic agents of different sizes formed by nonresorbable hydrogels and loaded with chemotherapy agents that ensure sustained and tumor-selective drug delivery as well as permanent embolization. ${ }^{10-13}$ Due to the properties of DEB, it is possible to reduce inadvertent portal vein embolization during TACE. Therefore, this paper aims to assess the safety and efficacy of DEB-TACE in HCC patients with APS.

\section{Materials and methods Patients}

This retrospective study was approved by the Institutional Review Board in accordance with approved guidelines of the Second Xiangya Hospital of Central South University and was compliant with Health Insurance Portability and Accountability Act. Written informed consent was obtained from all patients.

A total of 69 patients with unresectable HCC accompanied by APS who underwent TACE between September 2010 and December 2016 were included in this retrospective study. Patients with Child-Pugh class C, with an Eastern Cooperative Oncology Group performance status (ECOG ps) >2, or who underwent any treatments other than TACE throughout the study period were excluded. Thus, the final study population consisted of 58 patients. Of the 58 patients, 26 patients underwent DEB-TACE treatment, and 32 patients underwent polyvinyl alcohol (PVA) plus TACE (PVA-TACE) treatment.

\section{Interventions}

The femoral artery was routinely catheterized after administration of local anesthesia. Celiac trunk arteriogram, superior mesenteric arteriogram, and indirect portogram were performed using a $4 \mathrm{~F}$ catheter to validate the variant hepatic arterial anatomy and evaluate the patency of the portal vein. A selective arteriogram was performed to demonstrate the tumor blood supply vessels and APSs. The APSs were classified into four grades according to the severity of shunts: 0 , no or limited shunt flow, less than that of the subsegmental portal vein; 1, shunt flow regurgitated into the segmental portal vein; 2, shunt flow regurgitated into the ipsilateral main portal vein of each lobe; and 3 , shunt flow regurgitated into the contralateral lobe and/or the main portal vein. ${ }^{9}$ As patients with a grade 0 APS have a similar treatment strategy to those with grade 1 APS in our institution, we combined the two grades into a single grade in the present study.

In the DEB-TACE group, patients were treated with DEB (CalliSpheres Beads, Jiangsu Hengrui Medicine Co. Ltd). CalliSpheres Beads are a type of DEB that have shown reliable drug delivery. ${ }^{14}$ The DEBs were generated as follows: 1. Up to $80 \mathrm{mg}$ of pirarubicin hydrochloride (Main Luck Pharmaceuticals Co., Ltd.) was dissolved in $20 \mathrm{mg} /$ $\mathrm{mL}$ sterile 5\% glucose solution; 2. The CalliSpheres Beads were mixed with pirarubicin hydrochloride solution; 3 . The mixture was incubated for 30 minutes until the solution became relatively light in color; 4. Finally, the DEB solution was well prepared. The sizes of DEBs used were variable (100-700 $\mu \mathrm{m})$. One way to estimate particle sizes is based on the APS grading. Grade 1 shunt: $100-300 \mu \mathrm{m}$ DEB; grade 2 shunt: 300 - 500 $\mu \mathrm{m}$ DEB; and grade 3 shunt: $500700 \mu \mathrm{m}$ DEB. With the slow injection of a mixture of DEBs and contrast agent, the target lesions were gradually embolized. The embolization was stopped when the target vessel flow significantly or stagnantly decreased compared to the initial flow, which was often determined by measuring the time it took to clear the contrast column (typically four heart beats). A repeated hepatic arteriogram was performed to assess the devascularization and the direction of portal flow after embolization. For the PVA-TACE group, patients were treated in two steps. The first step was performed with PVA to embolize the shunt. The size of PVA particles varied from 180 to $710 \mu \mathrm{m}$. The choice of PVA size determination was similar to that of DEB size. When the APS showed complete occlusion by PVA embolization, cTACE with oil emulsion was performed as the second step. Oil emulsion was created using the oil-in-water technique by mixing $9 \mathrm{~mL}$ of iodized oil (Lipiodol, Guerbet Group) in a 3:1 ratio with $3 \mathrm{~mL}$ of $0.9 \%$ saline solution containing the drug cocktail composed of $40 \mathrm{mg}$ of dissolved pirarubicin. The endpoint of embolization in the PVA-TACE group was the same as that in the DEB-TACE group.

\section{Follow-up}

Follow-up was performed by outpatient clinic or telephone interviews until death or until the end of the study period (June 30, 2018). Unenhanced and contrast-enhanced computed tomography $(\mathrm{CT})$ or magnetic resonance (MR) was performed 1-3 months after the initial intervention and then at approximately 2-month intervals. Follow-up intervention was performed when there was viable tumor on the followup images according to the modified Response Evaluation Criteria in Solid Tumors (mRECIST). 


\section{Efficacy assessment}

Treatment efficacy was assessed by the improvement in APS and by the tumor response. The improvement in APS grade was defined as the postprocedural grade decreasing by at least one level compared to the preprocedural status. Tumor response on contrast-enhanced CT/MR was assessed by mRECIST based on the longest dimension of the viable tumor at 1-3 months after the first TACE session.

\section{Safety assessment}

Procedure-related toxicity was recorded and graded in both groups according to the National Cancer Institute Common Terminology Criteria for Adverse Events version 5.0 (NCI CTCAE v5.0). Procedure-related toxicity was divided into clinical toxicity and laboratory toxicity, which were assessed within 1 month after the procedure.

\section{Statistical analyses}

Continuous data are expressed as mean \pm SD. All statistical analyses were conducted using commercially available software (SPSS version 20, International Business Machines Corporation), and $P<0.05$ was considered statistically significant. For categorical variables, such as gender, tumor response, ECOG ps, Child-Pugh class, and the presence or absence of ascites, portal vein tumor thrombus (PVTT), and toxicities were compared between the two groups using a chi-squared test or Fisher's exact test (if appropriate). For noncategorical variables, such as age, serum albumin level, tumor size, and serum total bilirubin level, the Mann-Whitney U test was used. Survival curves were calculated using the Kaplan-Meier method, and the log-rank test was used to compare the survival rates between the two groups. To evaluate the prognostic factors in the DEB-TACE group, univariate analysis was performed to select explanatory variables, and potential prognostic factors were evaluated using a multivariate Cox regression model.

\section{Results}

\section{Patient characteristics}

Of the 58 patients, 57 patients had a history of cirrhosis, and 1 patient was noncirrhotic. The etiologies of liver cirrhosis were hepatitis B infection ( $n=49)$, hepatitis $C$ infection $(n=5)$, and alcoholic abuse $(n=3)$. The diagnosis of HCC was based on pathology (biopsy, $\mathrm{n}=6$ ) or on the American Association for the Study of Liver Practice Guidelines ( $\mathrm{n}=52)$. The clinical findings of the two groups are summarized in Table 1, and there were no significant differences in the baseline data between the two groups.

\section{Efficacy assessment}

APS was improved in all 58 patients after initial embolization. However, three patients in the DEB-TACE group and two patients in the PVA-TACE group showed recurrence of the shunt during follow-up, and there was no significant difference in the rate of shunt recurrence between the two groups $(P=0.648)$. For the evaluation of tumor response, the DEB-TACE group showed partial response (PR) in 8 $(30.8 \%, 8 / 26)$ patients, stable disease (SD) in $10(38.4 \%$, $10 / 26)$ patients, and progressive disease (PD) in $8(30.8 \%$, 8/26) patients according to the mRECIST criteria (Figure 1). In the PVA-TACE group, $4(12.5 \%, 4 / 32)$ patients showed PR, $8(25.0 \%, 8 / 32)$ patients had SD, and $20(62.5 \%, 20 / 32)$ patients had PD. Although the objective response rate did not differ between the two groups $(P=0.088)$, patients treated

Table I Clinical findings of two groups

\begin{tabular}{|l|l|l|l|}
\hline Characteristic & DEB-TACE group & PVA-TACE group & P-value \\
\hline Age (years) & $52.8 \pm 9.9$ & $54.3 \pm 12.0$ & 0.617 \\
Sex (male/female, \%) & $22 / 4,84.6 / 15.4$ & $27 / 5,84.4 / 15.6$ & 1.000 \\
Tumor size (mm) & $97.2 \pm 38.2$ & $108.1 \pm 59.0$ & 0.421 \\
Albumin (g/L) & $34.2 \pm 3.5$ & $35.8 \pm 3.9$ & 0.114 \\
Bilirubin (umol/L) & $17.8 \pm 7.7$ & $17.3 \pm 9.2$ & 0.854 \\
Child-Pugh class (A/B, \%) & $20 / 6,76.9 / 23.1$ & 0.347 \\
PVTT (yes/no, \%) & $12 / 14,46.1 / 53.9$ & $21 / 11,65.6 / 34.4$ & 0.672 \\
Ascites (yes/no, \%) & $5 / 21,19.2 / 80.8$ & $13 / 19,40.6 / 59.4$ & 0.299 \\
APS grading (I/2/3, \%) & $12 / 10 / 4,46.1 / 38.5 / 15.4$ & $10 / 22,31.2 / 68.8$ & 0.730 \\
BCLC staging (B/C, \%) & $13 / 13,50.0 / 50.0$ & $11 / 15 / 6,34.4 / 46.9 / 18.7$ & 0.813 \\
ECOG ps (0/I/2, \%) & $15 / 17,46.9 / 53.1$ & 0.400 \\
Distant metastasis (yes/no, \%) & $10 / 16 / 6,31.2 / 50.0 / 18.8$ & 0.205 \\
\hline
\end{tabular}

Abbreviations: APS, arterioportal shunt; BCLC, Barcelona Clinic Liver Cancer; DEB-TACE, transarterial chemoembolization with drug-eluting beads; ECOG ps, Eastern Cooperative Oncology Group performance status; PVA-TACE, polyvinyl alcohol plus transarterial chemoembolization; PVTT, portal vein tumor thrombus. 
with DEB-TACE showed a better disease control rate than did those treated with PVA-TACE $(P=0.016)$. The efficacy assessment results of the two groups are presented in Table 2.

\section{Survival}

The median survival times were 346 and 274 days in the DEB-TACE group and PVA-TACE group, respectively. The survival rates in the DEB-TACE group were $84.6 \%, 50.0 \%$, and $11.5 \%$ at 6,12 , and 24 months, respectively, while in the PVA-TACE group, the survival rates were $81.3 \%, 21.9 \%$, and $3.1 \%$ at 6,12 , and 24 months, respectively (Figure 2). There was no significant difference in survival rates between the DEB-TACE group and the PVA-TACE group $(P=0.081)$.

For the evaluation of prognostic factors in the DEBTACE group, univariate analysis showed that tumor response
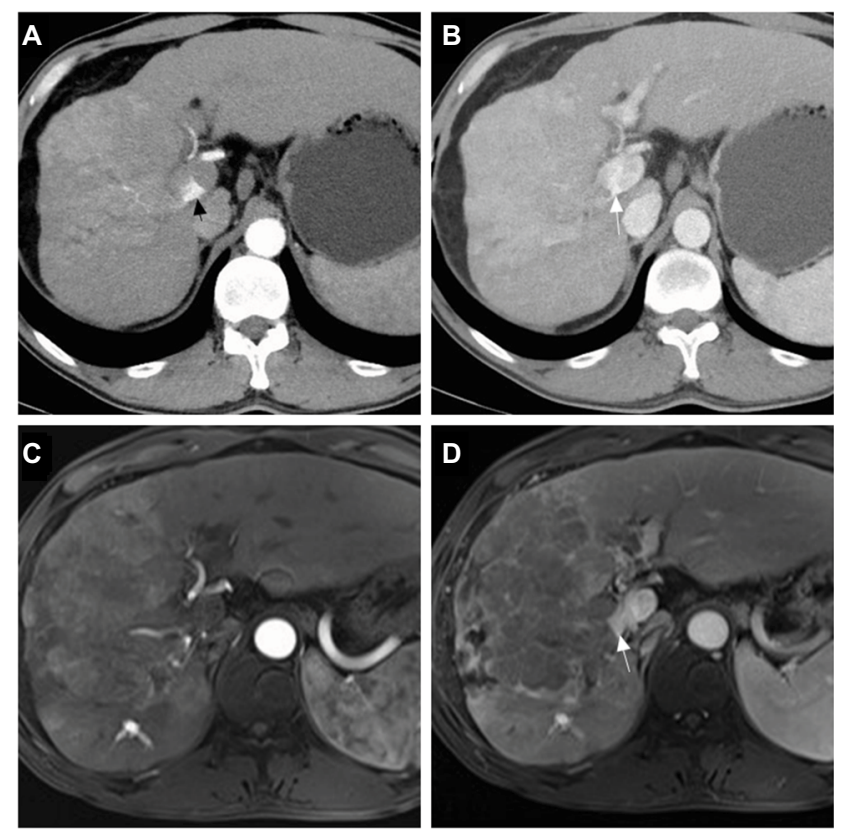

Figure I Tumor response of patients in the group with transarterial chemoembolization with drug-eluting beads.

Notes: A 48-year-old male with hepatocellular carcinoma and grade 3 arterioportal shunt: contrast-enhanced computed tomography scan showed a hepatic arterial phase hyperattenuation lesion with a maximal diameter of $106.5 \mathrm{~mm}$, the arterial flow was drained into the main portal vein (black arrow) $(\mathbf{A})$, and the portal venous phase showed tumor thrombus (white arrow) (B); a follow-up magnetic resonance scan was performed after 3 months, showing that the maximal diameter of the tumor was increased to $151.8 \mathrm{~mm}$ and indicating a tumor response of progression disease, the hepatic arterial phase (C), and the portal venous phase (D).
$(P=0.004)$, APS grade $(P=0.003)$, serum total bilirubin $(P=0.001)$, and tumor size $(P=0.013)$ were significantly correlated with survival. Multivariate survival analysis showed that (a) tumor response $(P=0.038, \mathrm{HR}=2.051,95 \%$ CI 1.039 , 4.047); (b) APS grade $(P=0.013, \mathrm{HR}=3.101,95 \%$ CI 1.268 , 7.587); and (c) serum total bilirubin ( $P=0.003, \mathrm{HR}=103.139$, $95 \%$ CI $4.658,2283.906)$ were potential prognostic factors (Table 3).

\section{Safety assessment}

The clinical and laboratory toxicities of the two groups are summarized in Table 4. Patients treated with DEB-TACE were significantly less likely to have fever $(P=0.048)$ and increased low-grade (grade $1-2)$ transaminases $(P=0.046)$ than were patients treated with PVA-TACE. In addition to fever and increased levels of transaminases, there were no significant differences in other clinical toxicities or laboratory toxicities between the two groups (all $P>0.05$ ). The most common clinical toxicities were abdominal pain and fever, and no patient developed hepatic failure or death within 1 month after the procedure in either group.

\section{Discussion}

Several studies have reported that APS is a poor prognostic factor in HCC patients because such patients are often associated with portal hypertension, refractory ascites, bleeding of esophageal and gastric varices, and hepatic encephalopathy. ${ }^{1-4}$ Due to the high risk of developing hepatic failure in patients with HCC and APS, cTACE is sometimes contraindicated. ${ }^{15}$ To the best of our knowledge, there is no standard treatment for the embolization of APS to date, and various embolic materials have been introduced, such as coils, gelatin sponges, absolute ethanol, glue, balloon-assisted embolization devices, and PVA particles. ${ }^{3,4,7-9,16-19}$ Unfortunately, the embolic agents mentioned above can only occlude a shunt, which may not achieve an ideal therapeutic effect for the corresponding tumor. It should be noted that there are at least two therapeutic goals for patients with HCC and APS. The first goal is shunt embolization, and the second is tumorous disease therapy. Because DEBs allow for sustained drug delivery

Table 2 Efficacy assessment of two groups

\begin{tabular}{|l|l|l|l|}
\hline Efficacy assessment & DEB-TACE group & PVA-TACE group & P \\
\hline Shunt recurrence (yes/no, \%) & $3 / 23,11.5 / 88.5$ & $2 / 30,6.3 / 93.7$ & 0.648 \\
Objective response (CR + PR/SD + PD, \%) & $8 / 18,30.8 / 69.2$ & $4 / 28,12.5 / 87.5$ & 0.088 \\
Disease control (CR + PR+SD/PD, \%) & $18 / 8,69.2 / 30.8$ & $12 / 20,37.5 / 62.5$ & 0.016 \\
\hline
\end{tabular}

Abbreviations: CR, complete response; DEB-TACE, transarterial chemoembolization with drug-eluting beads; PD, progression disease; PR, partial response; PVA-TACE, polyvinyl alcohol plus transarterial chemoembolization; SD, stable disease. 


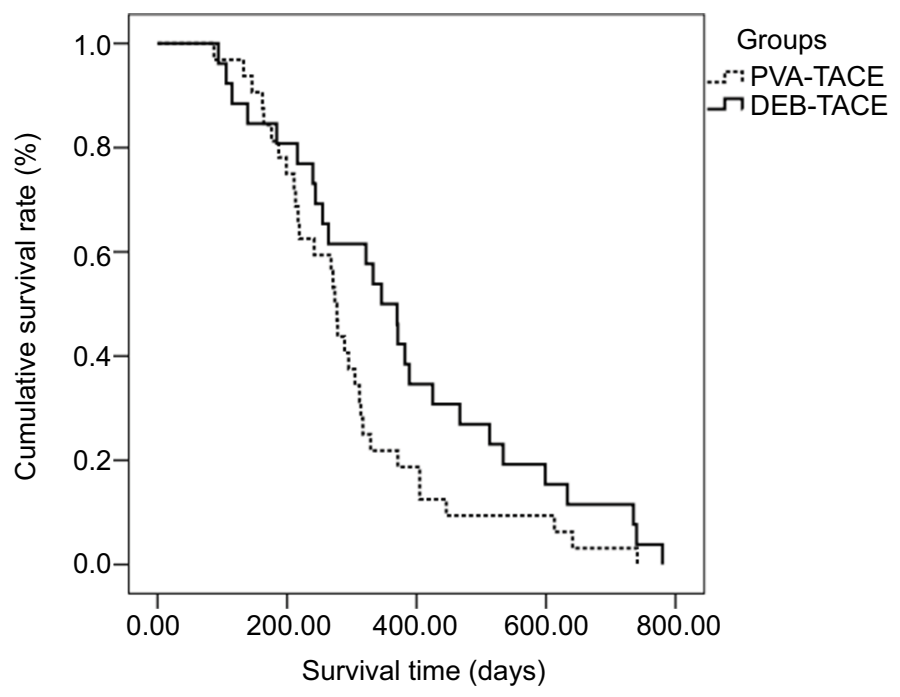

Figure 2 The overall survival of patients in the DEB-TACE group and PVA-TACE group.

Abbreviations: DEB-TACE, transarterial chemoembolization with drug-eluting beads; PVA-TACE, polyvinyl alcohol plus transarterial chemoembolization.

Table 3 Univariate and multivariate analyses of survival in DEB-TACE group

\begin{tabular}{|c|c|c|c|c|c|c|}
\hline \multirow[t]{2}{*}{ Variables } & \multirow[t]{2}{*}{ Categories } & \multicolumn{3}{|l|}{ Univariates } & \multicolumn{2}{|l|}{ Multivariates } \\
\hline & & Median OS (days) & $\chi^{2}$ & $\mathbf{P}$ & HR (95\% Cl) & $P$-value \\
\hline \multirow[t]{2}{*}{ Sex } & Male & 346 & 0.018 & 0.893 & & \\
\hline & Female & 322 & & & & \\
\hline \multirow[t]{2}{*}{ Child-Pugh Class } & A & 370 & 1.536 & 0.215 & & \\
\hline & B & 244 & & & & \\
\hline \multirow[t]{3}{*}{ Tumor response } & PR & 534 & 11.252 & 0.004 & 2.051 (1.039-4.047) & 0.038 \\
\hline & SD & 346 & & & & \\
\hline & PD & 240 & & & & \\
\hline \multirow[t]{3}{*}{ APS grade } & 1 & 425 & II.442 & 0.003 & 3.101 (1.268-7.587) & 0.013 \\
\hline & 2 & 346 & & & & \\
\hline & 3 & 184 & & & & \\
\hline \multirow[t]{2}{*}{ Ascites } & Absence & 370 & 0.805 & 0.369 & & \\
\hline & Presence & 346 & & & & \\
\hline \multirow[t]{2}{*}{ PVTT } & Absence & 370 & 1.916 & 0.166 & & \\
\hline & Presence & 333 & & & & \\
\hline \multirow[t]{3}{*}{ ALB } & $>35$ & 322 & 2.851 & 0.240 & & \\
\hline & $28-35$ & 346 & & & & \\
\hline & $<28$ & 371 & & & & \\
\hline \multirow[t]{2}{*}{ Total bilirubin } & $<34$ & 370 & 11.266 & 0.001 & $103.139(4.658-2283.906)$ & 0.003 \\
\hline & $34-51$ & 106 & & & & \\
\hline \multirow[t]{3}{*}{ ECOG ps } & 0 & 389 & 1.530 & 0.465 & & \\
\hline & 1 & 322 & & & & \\
\hline & 2 & 346 & & & & \\
\hline \multirow[t]{2}{*}{ Tumor size } & $<50 \mathrm{~mm}$ & 534 & 6.227 & 0.013 & & \\
\hline & $\geq 50 \mathrm{~mm}$ & 322 & & & & \\
\hline
\end{tabular}

Abbreviations: ALB, albumin; APS, arterioportal shunt; DEB-TACE, transarterial chemoembolization with drug-eluting beads; ECOG ps, Eastern Cooperative Oncology Group performance status; OS, overall survival; PD, progression disease; PR, partial response; PVTT, portal venous tumor thrombus; SD, stable disease.

and simultaneous permanent embolization, ${ }^{20-22}$ we believe that DEBs are an appropriate embolic agent for such patients.

The present study revealed that patients treated with DEBTACE had better disease control than those treated with PVA-
TACE. In previous studies, Liu et $\mathrm{al}^{8}$ and Kim et $\mathrm{al}^{16}$ showed that HCC patients with APS might benefit from PVA-TACE. However, the efficacy of PVA-TACE for such patients must be confirmed. PVA is a permanent particle embolic agent 
Table 4 Safety assessment of two groups

\begin{tabular}{|c|c|c|c|}
\hline Safety assessment & DEB-TACE group & PVA-TACE group & P-value \\
\hline \multicolumn{4}{|l|}{ Clinical toxicity (grade I-2) } \\
\hline Fever (yes/no, \%) & $16 / 10,61.5 / 38.5$ & $27 / 5,84.4 / 15.6$ & 0.048 \\
\hline Abdominal pain (yes/no, \%) & $22 / 4,84.6 / 15.4$ & $28 / 4,87.5 / 12.5$ & 1.000 \\
\hline Vomiting (yes/no, \%) & $3 / 23,11.5 / 88.5$ & $5 / 27,15.6 / 84.4$ & 1.000 \\
\hline \multicolumn{4}{|l|}{ Clinical toxicity (grade 3-4) } \\
\hline GI bleeding (yes/no, \%) & $\mathrm{I} / 25,3.8 / 96.2$ & $0 / 32,0 / 100$ & 0.448 \\
\hline Unbearable pain or fever (yes/no, \%) & $5 / 21,19.2 / 80.8$ & $8 / 24,25.0 / 75.0$ & 0.600 \\
\hline Hepatic failure (yes/no, \%) & $0 / 26,0 / 100$ & $0 / 32,0 / 100$ & NA \\
\hline \multicolumn{4}{|l|}{ Laboratory toxicity (grade I-2) } \\
\hline Increased transaminases (yes/no, \%) & $12 / 14,46.2 / 53.8$ & $23 / 9,71.9 / 28.1$ & 0.046 \\
\hline Increased bilirubin (yes/no, \%) & $6 / 20,23.1 / 76.9$ & $7 / 25,26.9 / 78.1$ & 0.913 \\
\hline Increased alkaline phosphatase (yes/no, \%) & $3 / 23,11.5 / 88.5$ & $3 / 29,9.4 / 90.6$ & 1.000 \\
\hline \multicolumn{4}{|l|}{ Laboratory toxicity (grade 3-4) } \\
\hline Increased transaminases (yes/no, \%) & $5 / 21,19.2 / 80.8$ & $7 / 25,21.9 / 78.1$ & 0.805 \\
\hline Increased bilirubin (yes/no, \%) & $0 / 26,0 / 100$ & I/3I, $3.1 / 96.9$ & 1.000 \\
\hline Increased alkaline phosphatase (yes/no, \%) & $0 / 26,0 / 100$ & $0 / 32,0 / 100$ & NA \\
\hline
\end{tabular}

Abbreviations: DEB-TACE, transarterial chemoembolization with drug-eluting beads; Gl, gastrointestinal; PVA-TACE, polyvinyl alcohol plus transarterial chemoembolization.

that may cause proximal embolization, thereby preventing Lipiodol from entering the tumor-feeding artery distally. Although the present study showed that there was no significant difference in survival rates between the two groups, the median survival was slightly longer in the DEB-TACE group than in the PVA-TACE group (346 days vs 274 days). In the literature, the 6-, 12-, and 24-month survival rates of patients with HCC and APS are reported to be $45 \%-91.7 \%$, $12 \%-85.7 \%$, and $8 \%-64.3 \%$, respectively. ${ }^{3,4,7-9,17-19}$ Among these studies, that reporting the highest survival rate was conducted by Murata et al ${ }^{19}(85.7 \%$ at 12 months and $64.3 \%$ at 24 months), in which the reported survival rates are higher than those obtained in the present study. One possible explanation might be due to the different study populations enrolled. In the study by Murata et al, patients with PVTT were excluded, while in our study, $43.1 \%$ (25/58) of the patients had PVTT, which might affect survival.

In the safety assessment, patients treated with DEB-TACE showed a lower incidence of fever and increased transaminases. Because of the slow and sustained drug delivery of DEB, the tolerance of the procedure should be better in DEBTACE than in PVA-TACE. Nevertheless, the most common toxicities were of low grade (grade 1-2), and no patient died or developed hepatic failure in either group, which indicated that both procedures were safe for the treatment of patients with HCC and APS.

Additionally, our study revealed that tumor response was an independent predictive factor of prognosis. A previous study showed that patients who responded to TACE exhibited improved survival, ${ }^{23}$ which was in accordance with our findings. APS grading was also a predictive factor of prognosis. Patients with higher APS grading may have more severe clinical symptoms, which will affect survival. ${ }^{24}$ The serum bilirubin level is another predictive factor. Several studies have reported that patients with high serum bilirubin levels are not suitable for TACE because such patients may not benefit from the procedure. ${ }^{25}$ When TACE is performed, these three factors should be taken into consideration.

\section{Limitations}

First, the present study had a retrospective design. A prospective study that compares the efficacy and safety between DEB-TACE and PVA-TACE for the treatment of patients with HCC and APS should be performed in the future. Second, the number of patients enrolled in the present study was too small, which may result in statistical bias.

\section{Conclusion}

DEB-TACE might be a feasible and safe method for treating patients with HCC and APS. The overall survival of such patients is associated with tumor response, APS grading, and serum bilirubin level.

\section{Acknowledgment}

This study was funded the by Hunan Provincial Natural Science Foundation of China (2018JJ6134).

\section{Disclosure}

The authors report no conflicts of interest in this work. 


\section{References}

1. Choi BI, Lee KH, Han JK, Lee JM. Hepatic arterioportal shunts: dynamic CT and MR features. Korean J Radiol. 2002;3(1):1-15.

2. Bookstein JJ, Cho KJ, Davis GB, Dail D. Arterioportal communications: observations and hypotheses concerning transsinusoidal and transvasal types. Radiology. 1982;142(3):581-590.

3. Vogl TJ, Nour-Eldin NE, Emad-Eldin S, et al. Portal vein thrombosis and arterioportal shunts: effects on tumor response after chemoembolization of hepatocellular carcinoma. World J Gastroenterol. 2011;17(10):1267-1275.

4. Huang MS, Lin Q, Jiang ZB, et al. Comparison of long-term effects between intra-arterially delivered ethanol and Gelfoam for the treatment of severe arterioportal shunt in patients with hepatocellular carcinoma. World J Gastroenterol. 2004;10(6):825-829.

5. Ngan H, Peh WC. Arteriovenous shunting in hepatocellular carcinoma: its prevalence and clinical significance. Clin Radiol. 1997;52(1):36-40.

6. Okuda K, Musha H, Yamasaki T, et al. Angiographic demonstration of intrahepatic arterio-portal anastomoses in hepatocellular carcinoma. Radiology. 1977;122(1):53-58.

7. Duan F, Bai Y, Cui L, Li X, Yan J, Zhu H. Transarterial embolization with N-butyl 2-cyanoacrylate for the treatment of arterioportal shunts in patients with hepatocellular carcinoma. $J$ Cancer Res Ther. 2017;13(4):631-635.

8. Liu QS, Mei QL, Li YH. Polyvinyl alcohol terminal chemoembolization for hepatocellular carcinoma with hepatic arteriovenous shunts: safety, efficacy, and prognostic factors. Eur J Radiol. 2017;89:277-283.

9. Shi HB, Yang ZQ, Liu S, et al. Transarterial embolization with cyanoacrylate for severe arterioportal shunt complicated by hepatocellular carcinoma. Cardiovasc Intervent Radiol. 2013;36(2):412-421.

10. Asayama Y, Okamoto D, Ushijima Y, et al. Predictors of therapeutic effect of transarterial chemoembolisation using drug-eluting beads for hepatocellular carcinoma. Clin Radiol. 2017;72(9):780-785.

11. Burrel M, Reig M, Forner A, et al. Survival of patients with hepatocellular carcinoma treated by transarterial chemoembolisation (TACE) using drug Eluting beads. Implications for clinical practice and trial design. J Hepatol. 2012;56(6):1330-1335.

12. Gaba RC, Emmadi R, Parvinian A, Casadaban LC. Correlation of doxorubicin delivery and tumor necrosis after drug-eluting bead transarterial chemoembolization of rabbit VX2 liver tumors. Radiology. 2016;280(3):752-761.

13. Sacco R, Bargellini I, Bertini M, et al. Conventional versus doxorubicin-eluting bead transarterial chemoembolization for hepatocellular carcinoma. J Vasc Interv Radiol. 2011;22(11):1545-1552.
14. Zhang S, Huang C, Li Z, et al. Comparison of pharmacokinetics and drug release in tissues after transarterial chemoembolization with doxorubicin using diverse lipiodol emulsions and CalliSpheres Beads in rabbit livers. Drug Deliv. 2017;24(1):1011-1017.

15. Yasui D, Murata $S$, Ueda $T$, et al. Novel treatment strategy for advanced hepatocellular carcinoma: combination of conventional transcatheter arterial chemoembolization and modified method with portal vein occlusion for cases with arterioportal shunt: a preliminary study. Acta Radiol. 2018;59(3):266-274.

16. Kim YJ, Lee HG, Park JM, et al. Polyvinyl alcohol embolization adjuvant to oily chemoembolization in advanced hepatocellular carcinoma with arterioportal shunts. Korean J Radiol. 2007;8(4):311-319.

17. Furuse J, Iwasaki M, Yoshino M, et al. Hepatocellular carcinoma with portal vein tumor thrombus: embolization of arterioportal shunts Radiology. 1997;204(3):787-790.

18. Zhou WZ, Shi HB, Liu S, et al. Arterioportal shunts in patients with hepatocellular carcinoma treated using ethanol-soaked gelatin sponge: therapeutic effects and prognostic factors. $J$ Vasc Interv Radiol. 2015;26(2):223-230.

19. Murata S, Tajima H, Nakazawa K, Onozawa S, Kumita S, Nomura K. Initial experience of transcatheter arterial chemoembolization during portal vein occlusion for unresectable hepatocellular carcinoma with marked arterioportal shunts. Eur Radiol. 2009;19(8):2016-2023.

20. Varela M, Real MI, Burrel M, et al. Chemoembolization of hepatocellular carcinoma with drug eluting beads: efficacy and doxorubicin pharmacokinetics. J Hepatol. 2007;46(3):474-481.

21. Poon RT, Tso WK, Pang RW, et al. A phase I/II trial of chemoembolization for hepatocellular carcinoma using a novel intra-arterial drugeluting bead. Clin Gastroenterol Hepatol. 2007;5(9):1100-1108.

22. Lammer J, Malagari K, Vogl T, et al; PRECISION V Investigators. Prospective randomized study of doxorubicin-eluting-bead embolization in the treatment of hepatocellular carcinoma: results of the precision $\mathrm{V}$ study. Cardiovasc Intervent Radiol. 2010;33(1):41-52.

23. Hu HT, Kim JH, Lee LS, et al. Chemoembolization for hepatocellular carcinoma: multivariate analysis of predicting factors for tumor response and survival in a 362-patient cohort. $J$ Vasc Interv Radiol. 2011;22(7):917-923.

24. Lee DH, Yoon HK, Song HY, Kim GC, Hwang JC, Sung KB. Embolization of severe arterioportal shunts in the patients with hepatocellular carcinoma: safety and influence on patient survival. $J$ Korean Radiol Soc. 1999;41(6):1117-1125.

25. Sieghart W, Hucke F, Peck-Radosavljevic M. Transarterial chemoembolization: modalities, indication, and patient selection. $J$ Hepatol. 2015;62(5):1187-1195.
Cancer Management and Research

\section{Publish your work in this journal}

Cancer Management and Research is an international, peer-reviewed open access journal focusing on cancer research and the optimal use of preventative and integrated treatment interventions to achieve improved outcomes, enhanced survival and quality of life for the cancer patient. The manuscript management system is completely online and includes

\section{Dovepress}

a very quick and fair peer-review system, which is all easy to use. Visit http://www.dovepress.com/testimonials.php to read real quotes from published authors. 